\title{
EL INVENTARIO DE HISTORIA DE PÉRDIDAS (IHP): PRESENTACIÓN Y UTILIDAD CLÍNICA
}

Chaurand, A.

Universitat de Barcelona

Consejo Nacional de Ciencia y Tecnología de México

Feixas, G.

Universitat de Barcelona

Neimeyer, R. A.

University of Memphis

\begin{abstract}
Contemporary theories of grief and bereavement emphasize the role of active adaptation to loss as a factor affecting a broadrange of psychosocial outcomes. Despite the substantial empirical research supporting this view, investigators typically have limited their attention to responses to the most recent losses suffered by the bereaved, rather than studying the legacy of adaptation to losses incurred across a lifetime. To provide this broader view, the authors present the History of Loss Inventory (HLI), which systematically assesses the way losses have been experienced by respondents and their perception of having adapted successfully or unsuccessfully to each. The HLI therefore could assist in psychological assessment and treatment planning, as well as permit the collection of relevant historical data in further research on bereavement.

Keywords: grief, bereavement, loss, depression.
\end{abstract}

Después de las reflexiones iniciales de Freud (1915), la psicología ha evolucionado sin que el tema del duelo (y la propia muerte) tuvieran mucho protagonismo. La obra de Bowlby (1973) contribuyó sustancialmente a destacar la importancia que el duelo merece en la compresión del sufrimiento humano y de la salud mental. Este proceso al que se enfrenta el ser humano tras sufrir una pérdida se vive como un episodio difícil y doloroso al que se tiene que hacer frente, por lo general, en varias ocasiones a lo largo de la vida.

En las últimas cuatro décadas, el desarrollo conceptual y metodológico sobre

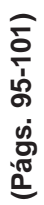
este tema ha sido muy importante, y las aportaciones realizadas en el desarrollo de la intervención psicológica en situaciones de duelo ha permitido ayudar de forma mucho más efectiva a las personas que las sufren. Actualmente es aún mayor el 
esfuerzo que se hace por estudiarlo, pues viviendo en una sociedad muy centrada en el día a día, con poco tiempo para compartir con los demás y para darse apoyo, el duelo es visto como una enfermedad, como "algo patético en lo que la persona no debería de perder el tiempo" tal como lo expresó en consulta un paciente. Además, en estos momentos en que las redes de apoyo con las que cuenta el ser humano son cada vez más impersonales, escasas y sin contacto real, el duelo ha mostrado mayores complicaciones, haciéndose presente de forma conflictiva en un número considerable de las personas que lo viven.

Como profesionales de la salud (y más como psicoterapeutas) es importante que al estar frente a un paciente haciendo una revisión sobre sucesos importantes de su vida, no dejemos de lado la evaluación de las pérdidas; éstas han marcado una parte importante en cada uno de nosotros ya desde el momento de nacer.

Al parecer la definición que más aceptación ha obtenido en cuanto a la pérdida es la propuesta por Harvey y Weber (1998) quienes la explican como cualquier daño en los recursos personales, materiales o simbólicos con los que hemos establecido un vínculo emocional.

El proceso que las pérdidas desencadenan en la persona, como lo mencionábamos anteriormente es el duelo, el cual según Payás (2010): "Es una experiencia de fragmentación de la identidad, producida por la ruptura de un vínculo afectivo: una vivencia multidimensional que afecta no solo a nuestro cuerpo físico y a nuestras emociones, sino también a nuestras relaciones con los demás y con nosotros mismos, a nuestras cogniciones, creencias y presuposiciones y a nuestro mundo interno existencial o espiritual" (p. 22).

Aunque algunas pérdidas se vivan como más significativas que otras, suelen venir acompañadas de dolor y sufrimiento y requieren un tiempo de recuperación y readaptación, o como sugiere Neimeyer (2007), de reconstrucción del mundo personal de significados que ha sido cuestionado por la propia pérdida. Para esta reconstrucción no existe un patrón definido, sino que cada persona con sus recursos personales debe encontrar la manera de hacer frente a la pérdida y todo lo que ello implica. El resultado más deseable de un proceso de duelo sano sería conseguir sacar algún provecho de la pérdida, como lo plantean Gil-Juliá, Bellver y Ballester (2008): "Es una experiencia inevitable que conlleva sufrimiento, pero también puede ser una oportunidad de crecimiento" (p. 103).

Es importante señalar que se puede vivir el proceso de duelo de forma satisfactoria y en la mayoría de ocasiones sin la necesidad de ayuda profesional. Bonanno y Kaltman (2001) señalan que la mayoría de las personas hacen frente a su pérdida y regresan a niveles de funcionamiento saludables durante el segundo año después de su ocurrencia. Complementando esto, Shear y cols. (2007) comentan que existe una minoría para la que la resolución del duelo agudo se ve bloqueada, lo que resulta en un duelo complicado (DC) que puede persistir por años e inclusive décadas.

La resolución satisfactoria o insatisfactoria del proceso de duelo depende en 
gran medida de diversos factores relacionados con la pérdida o mediadores de ésta (Worden, 2004): cómo era la persona, antecedentes históricos, variables de personalidad, edad y género, estresantes actuales, etc.

En general, la pérdida de un hijo, un esposo/a o un hermano (relaciones de primer grado) son consideradas más serias e inquietantes que las de relaciones de segundo grado (p. ej., abuelos, tíos, etc.) y que otras pérdidas. Pero hay siempre grandes diferencias individuales en función de cuando sucedió la pérdida, cómo sucedió (de forma violenta vs. natural, repentina vs. anticipada), las características de afrontamiento de la propia persona (segura vs. insegura), etc., éstos son factores relevantes para determinar el nivel de la sintomatología depresiva o del duelo, siendo estas dos formas de sufrimiento diferentes la una de la otra.

La respuesta natural a la pérdida que constituye el duelo está formada por un conjunto de reacciones a nivel cognitivo, conductual, emocional, etc., las cuales en ocasiones pueden ser semejantes a la sintomatología presentada en el Trastorno Depresivo Mayor (TDM) por lo que resulta, en la práctica, difícil de distinguir.

Se han llevado a cabo varios trabajos en los cuales se presentan críticas consistentes ante el criterio de exclusión del duelo en el DSM-IV-TR, con la finalidad de proponer la posible inclusión del diagnóstico de Duelo Complicado en el próximo DSM-V, así como también la de un apartado que especifique la sintomatología que puede presentarse debido a la reacción conocida como duelo normal (Lamb, Pies y Zisook, 2010; Zisook, Shear y Kendler, 2007; Zisook y Shear, 2009; Zisook, Reynolds, Pies, Simon, Lebowitz, Madowitz et al., 2010; Zisook, Simon, Reynolds, Pies, Lebowitz, Tal Young et al., 2010) o duelo agudo (Shear y Mulhare, 2008).

Tanto en el apartado de exclusión del DSM-IV-TR como en las propuestas mencionadas, el duelo es entendido únicamente como una reacción ante la muerte de un ser querido. Sin embargo, algunos investigadores (p. ej., Wakefield, Schmitz, First y Horwitz, 2007) consideran que deberían tenerse en cuenta también otros tipos de pérdidas.

Con la finalidad de estudiar con cierto detalle la historia de pérdidas de pacientes depresivos, y con la intención de poder investigar la relación entre duelo y depresión buscamos algún instrumento que nos permitiera conocer la historia de pérdidas que la persona presenta y su percepción sobre el hecho de haberlas superado o no. Pero no nos fue posible encontrar dicho instrumento ni tampoco investigaciones que evalúen la historia de las diversas pérdidas a lo largo de la vida de una persona. Algo que si encontramos fueron diversos tests adecuados para evaluar solamente los factores relacionados con el duelo por la muerte de un ser querido, como por ejemplo el Grief Evaluation Measure (GEM, Jordan, Baker, Matteis, Rosenthal y Ware, 2005). Encontramos también que existen herramientas de evaluación específicas para muerte perinatal, para cuidadores de pacientes con demencia, etc. (véase Neimeyer, Hogan y Laurie, 2009, para una revisión) pero 
ninguno que investigara la adaptación a los cambios que acompañan a una pérdida distinta a la de un ser querido.

Consideramos de gran importancia que los profesionales de la salud llevemos a cabo evaluaciones más completas y personalizadas. Con este propósito, y teniendo en cuenta algunas publicaciones anteriores sobre instrumentos de medición relacionados con el duelo y la pérdida (Neimeyer y Hogan, 2001; Neimeyer, et al., 2009) desarrollamos el Inventario de Historia de Pérdidas (IHP), un instrumento que nos permite obtener de forma breve una descripción de la historia de pérdidas del sujeto, detectar factores de riesgo o de complicación en el duelo y conocer la percepción que el paciente tiene tanto de la superación de su pérdida como de su sintomatología depresiva. Esto permitirá, en conjunto con lo que el profesional pueda detectar por medio de la evaluación clínica y de otros tests especializados, poder lograr un diagnóstico adecuado y planificar un tratamiento conveniente.

\section{El Inventario de Historia de Pérdidas (IHP)}

Es un inventario breve (véase anexo) en el que se pide al sujeto que haga una lista de las pérdidas significativas que ha vivido a lo largo de su vida. Para cada pérdida se le pregunta el tiempo transcurrido desde que ocurrió, la forma en que sucedió, y si considera haberla superado. Finalmente, se le pide que conteste afirmativa o negativamente si cree que alguna de estas pérdidas es el origen de su sintomatología depresiva o bien si considera que no presenta este tipo de síntomas.

Una aplicación interesante del IHP sería estudiar la relación que presenta con otros tests como el Inventario de Duelo Complicado (adaptación al castellano de Limonero, Lacasta, García, Maté y Prigerson, 2009) versión en español del Inventory of Complicated Grief (Prigerson, Maciejewsky, Reynolds III, Bierha1s, Newsom, Fasiczka, et al., 1995) para estimar el valor predictivo que pudiera presentar el IHP ante el diagnóstico de DC.

Aunque no consideramos necesario preguntar en esta breve evaluación por la red de apoyo del paciente, ni por el tipo de afrontamiento que ha presentado frente a pérdidas anteriores (factores evaluados por el GEM), creemos que es interesante tomar en cuenta esto a la hora de planificar el tratamiento, así como hacer una investigación más exhaustiva de aquellos aspectos fundamentales que a nivel personal podrían estar afectando la superación de la pérdida en la persona (GilJulia, et al., 2008).

Finalmente, destacar la necesidad de seguir investigando en los ámbitos del duelo y la depresión, así como llevar a cabo siempre una buena evaluación, completa y detallada. Con esto, podremos realizar un diagnóstico más preciso y otorgaremos a nuestros pacientes un mejor tratamiento. En la actualidad existen algunos tratamientos propuestos para el duelo complicado que están siendo puestos a prueba (Payás, 2010; Shear, Frank, Houck y Reynolds, 2005; Worden, 2004). 


\section{ANEXO \\ INVENTARIO DE HISTORIA DE PÉRDIDAS (IHP)}

Nombre:

Situación personal:

(Soltero, casado, viviendo en pareja, etc.)
Edad:

Estudios:
Hombre/Mujer

Con las siguientes preguntas se pretende de forma breve y detallada hacer un recuento sobre las pérdidas que la persona ha vivido a lo largo de su proceso vital.

1.- Asegúrese de leer con atención cada pregunta y responder de forma concreta. Es importante que mencione solamente las pérdidas que considere más significativas para usted (no es necesario rellenar todos los espacios, ni tampoco limitarse a ellos). (Ejemplos: muertes o separaciones de seres queridos, mascotas, bienes materiales, partes corporales, salud, proyectos personales, etc.)

\begin{tabular}{|l|l|l|l|l|}
\hline & $\begin{array}{l}\text { Nombre o } \\
\text { descripción de } \\
\text { la pérdida }\end{array}$ & $\begin{array}{l}\text { ¿Hace cuánto } \\
\text { sucedió? } \\
\text { (Especificar en } \\
\text { meses o años) }\end{array}$ & $\begin{array}{l}\text { ¿Como sucedió? (Por } \\
\text { enfermedad, acciden- } \\
\text { te, suicidio, de forma } \\
\text { esperada o inespera- } \\
\text { da, etc.) }\end{array}$ & $\begin{array}{l}\text { ¿Considera usted } \\
\text { haber superado esta } \\
\text { pérdida? }\end{array}$ \\
\hline a) & & & & \\
\hline b) & & & & \\
\hline c) & & & & \\
\hline d) & & & & \\
\hline e) & & & & \\
\hline f) & & & & \\
\hline g) & & & & \\
\hline h) & & & & \\
\hline i) & & & & \\
\hline j) & & & & \\
\hline
\end{tabular}

2.- Si presenta usted alguna sintomatología depresiva en la actualidad: ¿Considera que ésta se presentó como resultado de alguna de las pérdidas mencionadas previamente?

$-\mathrm{Si}$ -No

- No considero que yo tenga síntomas depresivos

3.- ¿De cuál(es)? 
Las teorías contemporáneas sobre el duelo enfatizan en el papel de la adaptación activa a la pérdida como un factor que afecta a un amplio rango de aspectos psicosociales. A pesar del considerable número de investigaciones empíricas que refuerzan este punto de vista, los investigadores comúnmente han limitado su atención a las respuestas a las pérdidas más recientes sufridas por las personas en duelo antes que estudiar su posible adaptación a las diversas pérdidas sufridas a lo largo de su historia vital. Para ello, los autores presentan el Inventario de Historia de Pérdidas (IHP), el cual evalúa sistemáticamente la forma en que las pérdidas han sido experimentadas por los sujetos y su percepción de haberse adaptado satisfactoria o insatisfactoriamente a cada una de ellas. El IHP puede ayudar en la evaluación psicológica y en la planificación del tratamiento, así como permitir la recolección de datos históricos relevantes para la investigación futura en temas de duelo y pérdida.

Palabras clave: duelo, pérdida, depresión.

\section{Referencias bibliográficas:}

BONANNO, G. Y KALTMAN, S. (2001). The varieties of grief experience. Clinical Psychlogy Review. 21(5), 705-734.

BOWLBY, J. (1973). Separation: Anxiety and anger. Attachment and loss. Londres. Hogarth Press.

FREUD, S. (1915). Mourning and melancholia. SE (14), 237-258.

GIL-JULiÁ, B., BELlVER, A. Y BALlESTER, R. (2008). Duelo: Evaluación, Diagnóstico y Tratamiento. Psicooncología. 5(1), 103-116.

HARVEY, J. Y WEBER, A. (1998). Why there must be a psychology ofloss. En H. J. Harvey (comp.), Perspectives of loss: A sourcebook. Filadelfia: Taylor \& Francis.

JORDAN, J., BAKER, J., MATTEIS, M., ROSENTHAL, S. Y WARE, E. (2005). The grief evaluation measure: An inicial validation study. Death Studies, 29(4), 301-332.

LAMB, K., PIES, R. Y ZISOOK, S. (2010) The bereavement exclusion for the diagnosis of major depression: To be or not to be. Psychiatry, 7(7), 19-25.

LIMONERO, J., LACASTA, M., GARCÍA, J., MATÉ, J Y PRIGERSON, H. (2009) Adaptación al castellano del inventario de duelo complicado. Medicina Paliativa, 16(5), 291-297.

NEIMEYER, R. (2007). Aprender de la pérdida. Una guía para afrontar el duelo. Barcelona: Bolsillo Paidós.

NEIMEYER, R., HOGAN, N. Y LAURIE, A. (2009). The measurement of grief: Psychometric considerations in the assesment of reactions to bereavement. En M. Stroebe, R. Hansson, H. Schut, y W. Stroebe (Comps.), Handbook of bereavement research and practice: Advances in theory and intervention (pp. 133-161). Washington: American Psychological Association.

PAYÁS, A. (2010). Las tareas del duelo: Psicoterapia del duelo desde un modelo integrativo relacional. Barcelona: Paidós.

PRIGERSON, H., MACIEJEWSKI, P., REYNOLDS III, C., BIERHALS, A., NEWSOM, J., FASICZKA, A., FRANK, E., DOMAN, J. Y MILLER, M. (1995). Inventory of complicated grief: A scale to measure maladaptive symptoms of loss. Psychiatry Research, 59(1-2), 65-79.

SHEAR, K., FRANK, E., HOUCK, P. Y REYNOLDS III, CHARLES, F. (2005). Treatment of complicated grief. A randomized controlled trial. Journal of the American Medical Association 293 (21), 2601-2608.

SHEAR, K., MONK, T., HOUCK, P., MELHEM, N., FRANK, E., REYNOLDS III, C. Y SILLOWASH, R. (2007). An attachment based model of complicated grief including the role of avoidance. European Archives of Psychiatry and Clinical Neurosciences, 257, 453-461.

WAKEFIELD, J.C., SCHMITZ, M.F., FIRST, M.B. Y HORWITZ, A.V. (2007). Extending the bereavement exclusion for major depression to other losses. Archivo General de Psiquiatria, 64, 433-440.

WORDEN, J.W. (2004). El tratamiento del duelo: asesoramiento psicológico y terapia. Barcelona: Paidós.

ZISOOK, S., SHEAR, K. Y KENDLER, K. (2007). Validity of the bereavement exclusion criterion for the diagnosis of major depressive episode. World Psychiatry, 6(2), 102-107.

ZISOOK, S. Y SHEAR, K. (2009). Grief and bereavement: what psychiatrists need to know. World Psychiatry, 8 (2), 67-74. 
ZISOOK, S., REYNOLDS III, C., PIES, R., SIMON, N., LEBOWITZ, B., MADOWITZ, J., TAL YOUNG, I. Y SHEAR, K. (2010). Bereavement, complicated grief and DSM, Part 1: Depression. Journal of Clinical Psychiatry 71(0), 1-2.

ZISOOK, S., SIMON, N., REYNOLDS III, C., PIES, R., LEBOWITZ, B., TAL YOUNG, I., MADOWITZ, J. Y SHEAR, K. (2010). Bereavement, complicated grief and DSM, Part 2: Complicated grief. Journal of Clinical Psychiatry 71(8), 1097-1098. 